\title{
Candida Species Causing Neonatal Septicaemia - Experience in a Tertiary Care Centre
}

\author{
Supriya Sneha Lakra' ${ }^{1}$, Vasant P. Baradkar ${ }^{2}$, Jayanthi S. Shastri ${ }^{3}$ Anuradha De ${ }^{4}$
}

\begin{abstract}
${ }^{1}$ Department of Microbiology, Topiwala National Medical College and BYL Nair Ch. Hospital, Mumbai, Maharashtra, India. ${ }^{2}$ Department of Microbiology, Topiwala National Medical College and BYL Nair Ch. Hospital, Mumbai, Maharashtra, India. ${ }^{3}$ Department of Microbiology, Topiwala National Medical College and BYL Nair Ch. Hospital, Mumbai, Maharashtra, India. ${ }^{4}$ Department of Microbiology, Topiwala National Medical College and BYL Nair Ch. Hospital, Mumbai, , Maharashtra, India.
\end{abstract}

\section{ABSTRACT}

\section{BACKGROUND}

Candida species is one of the most common causes of bloodstream infections among neonates and accounts for $9-13 \%$ of such infections. Non-albicans Candida have emerged as important opportunistic pathogens, notably C. tropicalis, C. glabrata, $C$. parapsilosis and $C$. krusei. With the introduction of antifungal agents, the causes of Candida infections shifted from an almost complete dominance of Candida albicans to the common involvement of Candida glabrata and other non-albicans Candida species.

\section{METHODS}

This prospective, microbiological observational study was conducted in a tertiary care hospital for one and a half years from July 2016 to December 2017. Blood samples of neonates collected into Bactec Peds Plus/F Culture vials of an automated blood culture system (Bactec 9120, Becton Dikinson, USA) from clinically suspected cases of neonatal septicaemia were subjected to culture. Detailed clinical history such as presence of respiratory distress, abdominal distension, lethargy, feed intolerance, failure to thrive, poor perfusion, history of convulsions, duration of NICU stay and antibiotic use was taken from the medical records. The Candida species isolated were identified using standard mycological techniques.

\section{RESULTS}

Out of the total 250 clinically suspected cases of neonatal septicaemia, fungal growth was positive in $30(12 \%)$ cases. Candida albicans accounted for $10 \%$ and non albicans Candida accounted for $90 \%$ of the fungal isolates. Candida glabrata (73.33\%) was the commonest species followed by Candida tropicalis $(16.67 \%)$ and Candida albicans (10\%). Failure to thrive (60\%), lethargy (50\%) and respiratory distress $(30 \%)$ were the most common clinical presentations seen, followed by feed intolerance (26.67\%), abdominal distension (13.33\%) and fever (10\%). Among the risk factors observed for neonatal candidemia, low birth weight $(76.67 \%)$ and prematurity $(73.33 \%)$ were commonest followed by broad spectrum antibiotic use (66.67\%), total parenteral nutrition (53.3\%), ventilator support (36.67\%) and indwelling catheters $(26.67 \%)$.

\section{CONCLUSIONS}

Non-albicans Candida has emerged as an important pathogen causing neonatal septicaemia. Fungal sepsis is more common among preterm, low birth weight infants, those with prolonged antibiotic use and those on total parenteral nutrition. Candida glabrata caused the highest number of cases of fungal septicaemia.

\section{KEY WORDS}

Neonatal Candidemia, Non-Albicans Candida, Candida Glabrata
Corresponding Author: Dr. Supriya Sneha Lakra, E-304, Prema Sadan CHS, Ratan Nagar Lane, Four Bungalows, Andheri West, Mumbai-400053, Maharashtra, India.

E-mail: lakra.supriya93@gmail.com

DOI: $10.14260 /$ jemds $/ 2020 / 9$

Financial or Other Competing Interests: None.

How to Cite This Article:

Lakra SS, Baradkar VP, Shastri JS. et al. Candida species causing neonatal septicaemia- experience in a tertiary care centre. J. Evolution Med. Dent. Sci. 2020;9(01):38-43, DOI: 10.14260/jemds/2020/9

Submission 12-11-2019,

Peer Review 19-12-2019,

Acceptance 27-12-2019,

Published 06-01-2020. 


\section{BACKGROUND}

Significance of Candida species in neonatal intensive care unit (NICU) is increasingly being recognized. Candida species is one of the most common causes of bloodstream infections among neonates and accounts for $9-13 \%$ of such infections. ${ }^{1}$ Historically Candida albicans has been the most frequently isolated species worldwide. Recently non- albicans Candida have emerged as important opportunistic pathogen, notably C. tropicalis, C. glabrata, C. parapsilosis and C. krusei.2,3,4 Candidemia is a significant cause of mortality and morbidity in neonates. ${ }^{5}$ Previous studies have suggested that possible risk factors such as very low birth weight (LBW), prematurity, prolonged antibiotic therapy, prolonged use of fat emulsions in total parenteral nutrition (TPN), use of artificial ventilation, presence of indwelling central venous catheters (CVC), and intensive care unit (ICU) stay have made neonates prone to candidemia. Preterm, very low birth weight (VLBW): $\leq 1500 \mathrm{~g}$; extremely LBW: $\leq 1000 \mathrm{~g}$; and critically ill infants are at highest risk of invasive Candida infections. The clinical manifestations are respiratory insufficiency, apnoea, bradycardia, feeding intolerance, temperature instability and abdominal distension. ${ }^{5}$ Colonization of skin and gastrointestinal tract is the first step in the pathogenesis of invasive candidiasis. ${ }^{6}$ Delay in recognition of Candida infections and in the initiation of appropriate antifungal therapy often leads to significant morbidity and mortality rates among high-risk infants.

The genus Candida encompasses more than 150 species, only a few of which cause disease in humans. With rare exceptions, the human pathogens are Candida albicans, Candida guilliermondii, Candida krusei, Candida parapsilosis, Candida tropicalis, Candida kefyr, Candida lusitaniae, Candida dubliniensis, and Candida glabrata.7,8 These organisms are found on inanimate objects, in foods, and on animals, and are normal commensals of humans. They inhabit the gastrointestinal tract (including the mouth and oropharynx), the female genital tract, and the skin in humans with introduction of antifungal agents, the causes of Candida infections shifted from an almost complete dominance of Candida albicans to the common involvement of Candida glabrata and the other species listed above. ${ }^{9}$ The NAC species now account for approximately half of all cases of candidemia and hematogenously disseminated candidiasis. Recognition of this change is clinically important, since the various species differ in susceptibility to the newer antifungal agents. In developed countries, where medical therapeutics are commonly used, Candida species are now among the most common nosocomial pathogens. ${ }^{9}$ There are multiple risk factors that are responsible for neonatal candidemia like preterm such as low birth weight $<1500 \mathrm{~g}$ and infection through vertical transmission from maternal flora or via horizontal transmission from hands of health care workers. The incidence and associated mortality due to candidemia can be influenced by several factors including characteristic of population at risk, standard of the health care facilities available, distribution of Candida species and prevalence of antifungal resistance. Systemic candidiasis in neonates is increasing in frequency especially since the survival of babies with low birth weight (LBW) has increased. ${ }^{6}$ Considering all these facts, the present study was conducted to find out the percentage of isolation of Candida species from clinically suspected cases of neonatal septicaemia; the underlying comorbid conditions in culture proven cases of candidemia and identify Candida species causing septicaemia in neonates.

\section{METHODS}

The study was a cross-sectional microbiological observational study carried out for one and a half year duration from July 2016 to December 2017. The study was conducted after approval from the Institutional Ethics Committee. Informed consent was taken from the parent (Of neonate) prior to the patient enrolment.

Blood samples of neonates collected into Bactec Peds plus/F culture vials of an automated blood culture system (Bactec 9120, Becton Dikinson, USA) from clinically suspected cases of neonatal septicaemia was subjected to culture. Detailed clinical history such as presence of respiratory distress, abdominal distension, lethargy, feed intolerance, failure to thrive, poor perfusion, history of convulsions, duration of NICU stay and antibiotic use was taken from the medical records. When pathogenic bacteria gain access into the bloodstream, they may cause overwhelming infection without much localization (septicaemia). Candidemia is defined as the presence of at least one positive blood culture containing pure growth of Candida species with supportive clinical features. ${ }^{10}$

Smears were prepared of the samples that flashed positive in the automated blood culture system. The positive samples were subcultured on $5 \%$ sheep blood agar plate and were kept for overnight incubation at $37^{\circ} \mathrm{C}$. Candidal growth was confirmed by gram staining of the smears prepared from growth on blood agar plate. Any growth indicated was subcultured on Sabouraud dextrose agar with chloramphenicol $(0.05 \%)$ and cycloheximide and incubated at $37^{\circ} \mathrm{C}$ and at room temperature. ${ }^{11}$ All the Candida isolates were subjected to germ tube test using normal human serum. Colonies were identified up to the species level on the basis of morphology on Corn meal agar (Dalmau method) and sugar assimilation pattern.

\section{Statistical Analysis}

For data analysis, frequency and percentages were calculated. The percentage of isolation of Candida species from the total number of clinically suspected cases of neonatal septicaemia was calculated. The underlying co-morbid conditions in the culture proven cases of candidemia were enlisted and frequency table was formulated. Candida species causing neonatal septicaemia were identified and frequency tables were formulated.

\section{RESULTS}

Out of the total 250 clinically suspected cases of neonatal septicaemia, growth was seen in $40(16 \%)$ of cases. Fungal growth was positive in $30(12 \%)$ cases, bacterial growth was seen in $10(4 \%)$ cases, $11(4.4 \%)$ showed contamination and 199 (79.6\%) did not show any growth. Candida albicans accounted for $10 \%$ and non albicans Candida accounted for 
$90 \%$ of the fungal isolates. Candida glabrata $(73.33 \%)$ was the commonest species followed by Candida tropicalis $(16.67 \%)$ among the fungal isolates. The bacterial growth constituted of Klebsiella pneumoniae 4 (1.6\%), methicillin resistant Staphylococcus aureus (MRSA) 3 (1.2\%), Enterococcus sp. 1 (0.4\%), Streptococcus sp. 1 (0.4\%) and Enterobacter $s p .1$ (0.4\%). Candida $s p$. were isolated from $30 / 40(75 \%)$ of positive cases while bacteria were isolated from 10/40 (25\%) of positive cases. Candida glabrata (73.33\%) was the commonest isolate followed by Candida tropicalis (16.67\%) and Candida albicans (10\%). The average gestational age was 32 weeks (30-39 weeks) and average birth weight was $1.77 \pm 0.615 \mathrm{~kg}(0.82-3 \mathrm{~kg})$. Among the risk factors observed for neonatal candidemia, low birth weight (76.67\%) and prematurity (73.33\%) were commonest followed by broad spectrum antibiotic use (66.67\%), total parenteral nutrition $(53.3 \%)$, ventilator support $(36.67 \%)$ and indwelling catheters (26.67\%). Failure to thrive (60\%), lethargy (50\%) and respiratory distress (30\%) were the most common clinical presentations seen, followed by feed intolerance (26.67\%), abdominal distension (13.33\%) and fever (10\%).
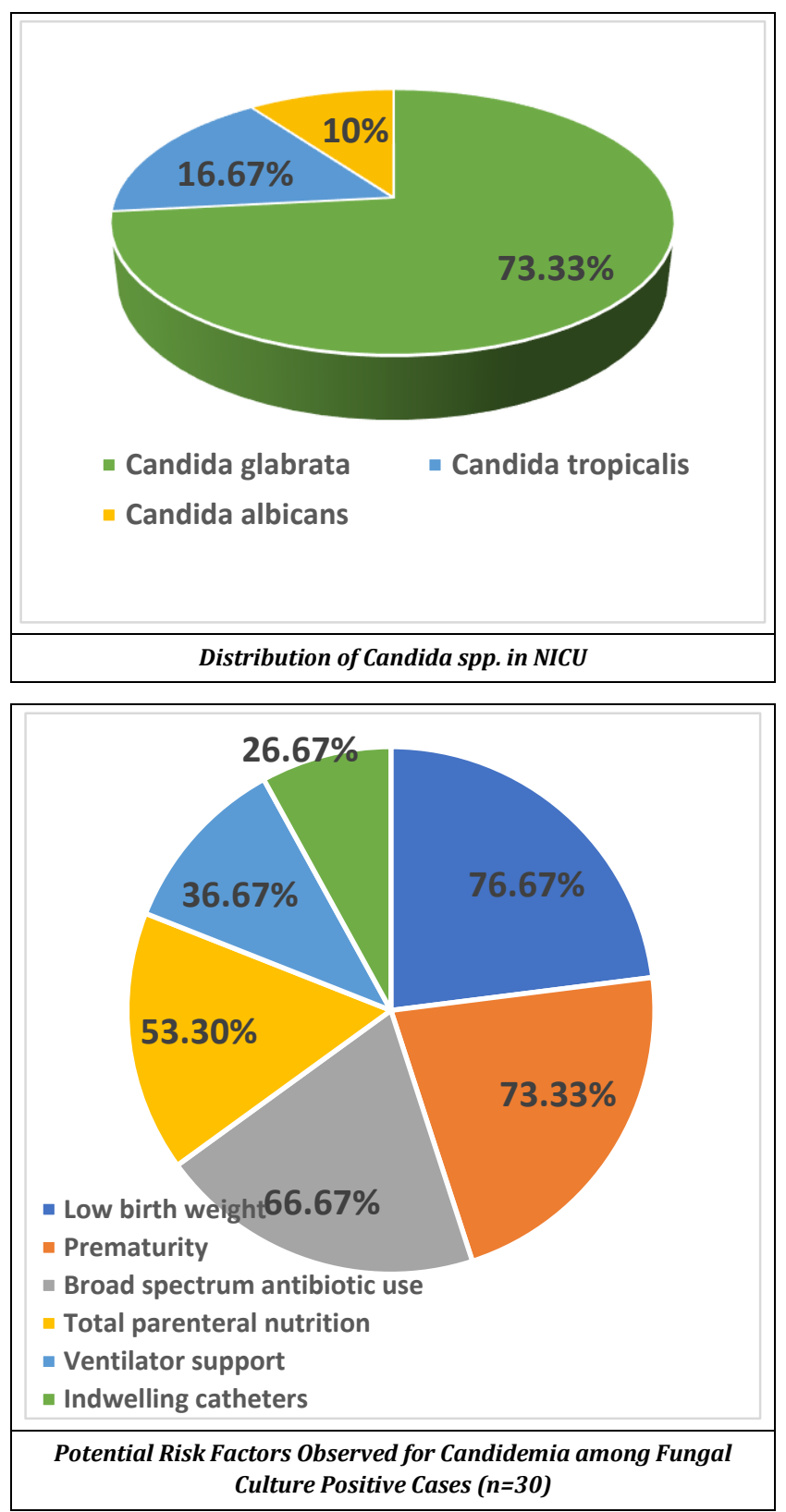

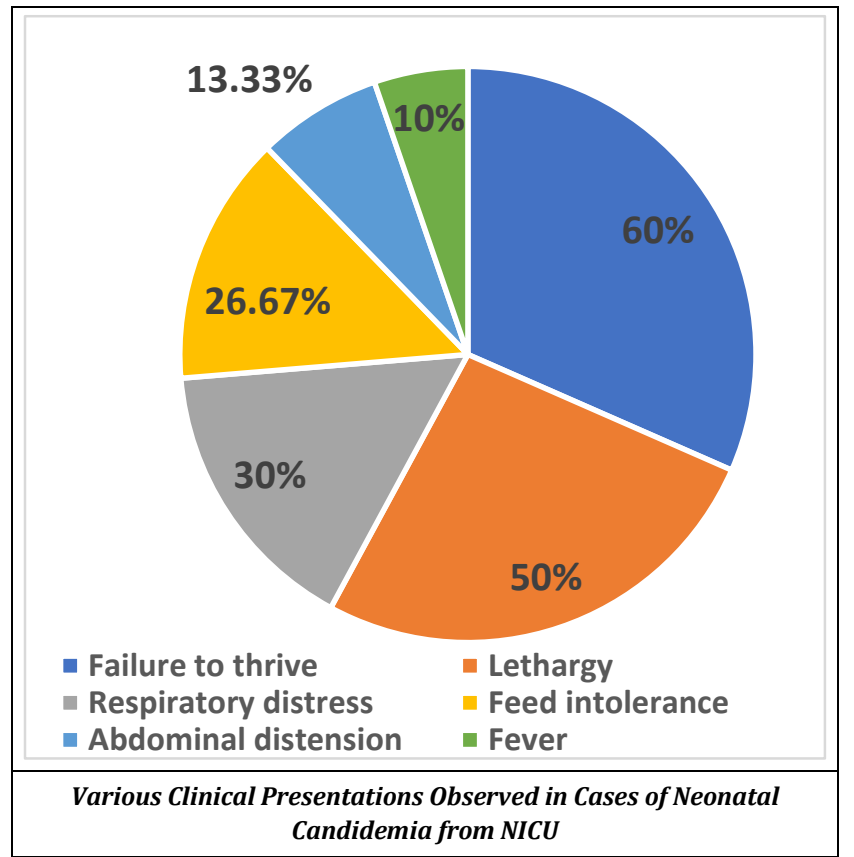

\begin{tabular}{|c|c|c|}
\hline Total & $\mathbf{2 5 0}$ & Percentage \\
\hline Growth & 40 & $16 \%$ \\
\hline Fungal Growth & 30 & $12 \%$ \\
\hline Bacterial Growth & 10 & $4 \%$ \\
\hline Contamination & 11 & $4.4 \%$ \\
\hline No Growth & 199 & $79.6 \%$ \\
\hline \multicolumn{3}{|c|}{ Culture Results } \\
\hline
\end{tabular}

\begin{tabular}{|c|c|c|}
\hline Growth & Number & Percentage \\
\hline Candida glabrata & 22 & $73.33 \%$ \\
\hline Candida tropicalis & 5 & $16.67 \%$ \\
\hline Candida albicans & 3 & $10 \%$ \\
\hline \multicolumn{2}{|r|}{ Distribution of Candida Species in NICU } \\
\hline
\end{tabular}

\begin{tabular}{|c|c|c|}
\hline Risk Factors & Number of Cases & Percentage \\
\hline Low birth weight & 23 & $76.67 \%$ \\
\hline Prematurity & 22 & $73.33 \%$ \\
\hline Broad spectrum antibiotic use & 20 & $66.67 \%$ \\
\hline Total parenteral nutrition & 16 & $53.3 \%$ \\
\hline Ventilator support & 11 & $36.67 \%$ \\
\hline Indwelling catheters & 8 & $26.67 \%$ \\
\hline Potential Risk Factors Observed for Candidemia \\
among the Fungal Culture Positive Cases (n=30)
\end{tabular}

\begin{tabular}{|c|c|c|}
\hline Sign/Symptoms & Number of Cases & Percentage \\
\hline Failure to thrive & 18 & $60 \%$ \\
\hline Lethargy & 15 & $50 \%$ \\
\hline Respiratory distress & 9 & $30 \%$ \\
\hline Feed intolerance & 8 & $26.67 \%$ \\
\hline Abdominal distension & 4 & $13.33 \%$ \\
\hline Fever & 3 & $10 \%$ \\
\hline \multicolumn{2}{|c|}{ Various Clinical Presentations Observed in } \\
Cases of Neonatal Candidemia (n=30) \\
\hline
\end{tabular}

\section{DISCUSSION}

The rate of isolation of Candida species from fungal culture positive isolates in our study is $12 \%$ which is comparable to the study done by Kumar et al ${ }^{12}$ in 2011 , in which the rate of isolation was $14.9 \%$. Various studies have been carried out over the last decade which showed the rate of isolation of Candida species as $19.14 \%$ (Baradkar et al, 2008), ${ }^{1} 30.1 \%$ (Sardana et al, 2012),13 20.39\% (Srinivas Rao et al, 2014),14 $32.26 \%$ (Wadile et al, 2015). ${ }^{6}$ 
Several studies have demonstrated the association of various risk factors with candidemia like low birth weight, preterm delivery, prolonged use of higher antibiotics like third generation cephalosporins and carbapenems, use of intravenous catheters, parenteral nutrition etc. ${ }^{15,6}$ These risk factors increase the susceptibility to infections because of the immaturity of the immune system and invasive medical equipment needed for improvement of the survival rate of the neonates. LBW, prematurity and use of broad spectrum antibiotics were the major risk factors implicated followed by total parenteral nutrition, ventilator support and indwelling catheters in the present study. This was in consistence with other studies done by Sardana et al, Juyal et al, Srinivas Rao et al and Wadile et al.13,6, 14,16

Failure to thrive (60\%), lethargy (50\%) and respiratory distress $(30 \%)$ were the most common clinical presentations seen, followed by feed intolerance (26.67\%), abdominal distension (13.33\%) and fever (10\%). Failure to thrive $(18 / 30)$ and lethargy $(15 / 30)$ were consistent clinical findings in our neonatal cases, similar to other studies. ${ }^{17,18,6,19}$ Respiratory distress $(74.55 \%)$ followed by failure to thrive and lethargy were most common clinical findings in a study by Sardana et al whereas Juyal et al showed failure to thrive (74.42\%) followed by abdominal distension and feed intolerance. ${ }^{13,16}$

Candida species are an increasingly common cause of neonatal sepsis and are responsible for considerable morbidity and mortality. ${ }^{20,21,17}$ Modern day neonatal care has definitely improved the survival rate of neonates but also increased the use of multiple invasive medical equipment, which has further enhanced the acquired sepsis among the newborns. Over the last two decades, non-albicans Candida (NAC) are accounting for a large burden of neonatal septicaemia.18,22,13 Agarwal and co-authors reported that 76 out of the 90 isolates were NAC. Yadav et al also observed $88.46 \%$ of the isolates belonged to non albicans group. ${ }^{20,23}$ In our study, non-albicans Candida bloodstream infections were commonest $(90 \%)$. This finding is consistent with other studies where non-albicans Candida spp. predominate in Asia, South Europe, South America and also in the subcontinent of India. ${ }^{17,24}$

C. glabrata was the commonest species isolated in $73.33 \%$ cases of candidemia followed by $C$. tropicalis (16.67\%) and C. albicans (10\%) in the present study. Whereas in a study by Yadav et al, ${ }^{20}$ the commonest species isolated was $C$. tropicalis $(26.92 \%)$. The rate of isolation of Candida glabrata $(73.33 \%)$ in the present study is quite high in comparison to other studies done over the last ten years. According to previous studies the rate of isolation of $C$. glabrata has been $61.22 \%$ (Baradkar et al, 2008), ${ }^{1} 19.69 \%$ (Kumar et al, 2011), ${ }^{2}$ 39\% (Sardana et al, 2012),13 13.50\% (Juyal et al, 2014),16 19.23\% (Srinivas Rao et al, 2014), ${ }^{14} 6.7 \%$ (Shrivastava et al, 2015), ${ }^{9} 10 \%$ (Wadile et al, 2015), ${ }^{6} 15.8 \%$ (Sil et al, 2017) ${ }^{25}$ and 33.3\% (Fu et al, 2017). ${ }^{26}$ Non-albicans Candida spp. are of special concern, due to their high virulence and low azole susceptibility characteristics, augmenting the high mortality rates. This rise is suggested to be a result of fluconazole prophylaxis given as a practice in many tertiary care centers.
In the present study, out of the total 250 clinically suspected cases of neonatal septicaemia, fungal growth was positive in $12 \%$ cases and bacterial growth was seen in $4 \%$ cases. The clinical manifestations of neonatal sepsis are indistinguishable in bacterial and fungal septicaemia. Thus it is important to identify the etiological agent in order to start appropriate treatment. $4.4 \%$ showed contamination which included micrococci and diphtheroids.

Probably infections due to Candida species are endogenous. It has been studied that about $10 \%$ of babies in the NICU get colonized in the first week of life and $64 \%$ babies get colonized by 4 weeks of hospital stay. The gastrointestinal tract is the first to become colonized though multiple sites may be involved.1,27 There is some evidence showing correlation between fungal colonization and invasive disease in very low birth weight, premature babies.1,27,28 Microorganisms including Candida spp. causing pneumonia acquired during labour and delivery may also act as source of candidemia. ${ }^{29}$ Various fungal agents colonize hospitalized infants, healthcare workers and visitors. Pathogenic organisms can be transmitted by direct contact or indirectly via contaminated instruments and intravenous fluids. ${ }^{27}$ This endogenous source, together with other predisposing factors as long-term antibiotics, catheterization, patients on ventilator, respiratory distress syndrome lead to candida septicaemia in NICU. ${ }^{1}$

Historically, Candida glabrata has been considered to be relatively nonpathogenic saprophyte of normal flora of healthy individuals rarely causing serious infections. ${ }^{30}$ However, following widespread and increased use of immunosuppressive therapy, broad spectrum antibiotic therapy, increased conditions causing compromise of the immune system, the frequency of mucosal as well as systemic infections caused by Candida glabrata has increased significantly.1,31,32,33,30 It is the only species of Candida that does not form pseudohyphae, it is found as blastoconidia (1-4 $\mu \mathrm{m})$, both as commensal and as pathogenic states. On Sabouraud's Dextrose agar, it forms glistening smooth, cream colored colonies, indistinguishable from Candida albicans but on Cornmeal agar it does not form pseudohyphae. It assimilates only glucose and trehalose. 30 Karen et al,29 compared Candida glabrata sepsis with other. According to this significant study, Candida glabrata sepsis occurred in infants with higher gestational periods (29.7 weeks as against 26.6 weeks in case of Candida albicans) and birth weight (Candida glabrata 1442 g, Candida albicans 931 g), but no such difference was observed in the present study.

The reasons behind the emergence of the species as predominant pathogen could be because of selection of lesser susceptible species due to frequent use of fluconazole as prophylaxis. ${ }^{4}$ The changing epidemiology of candidaemia, therefore, highlights the need for close monitoring of Candida species distribution and susceptibility in order to optimise therapy and outcome. We should also develop guidelines for empiric therapy based on the epidemiology of India.34 Understanding the mechanisms of innate and acquired resistance may facilitate the development of new targets for antifungal agents. More and more comprehensive studies of its epidemiology, pathogenesis, and resistance are needed to control the infections by Candida glabrata properly. ${ }^{1}$ 


\section{CONCLUSIONS}

In our study, among the two hundred and fifty (250) clinically suspected cases of neonatal septicaemia, $12 \%$ were fungal culture positive. Amongst these, Candida glabrata (73.33\%) was the commonest pathogen isolated, followed by Candida tropicalis (16.67\%) and Candida albicans (10\%). Nonalbicans Candida has emerged as an important pathogen causing neonatal septicaemia. Fungal sepsis is more common among preterm, low birth weight infants, those with prolonged antibiotic use and in those on total parenteral nutrition. Candida glabrata caused the highest number of cases of fungal septicaemia.

\section{REFERENCES}

[1] Baradkar VP, Mathur M, Kumar S, et al. Candida glabrata: emerging pathogen in neonatal sepsis. Annals of Tropical Medicine and Public Health 2008;1(1):5-8.

[2] Goel N, Ranjan PK, Aggarwal R, et al. Emergence of nonalbicans Candida in neonatal septicaemia and antifungal susceptibility: experience from a tertiary care center. Journal of Laboratory Physicians 2009;1(2):53-5.

[3] Oberoi JK, Wattal C, Goel N, et al. Non-albicans Candida species in bloodstream infections in a tertiary care hospital at New Delhi, India. The Indian Journal of Medical Research 2012;136(6):997-1003.

[4] Agarwal J, Bansal S, Malik GK, et al. Trends in neonatal septicaemia: emergence of non-albicans Candida. Indian Pediatrics 2004;41(7):712-5.

[5] Narain S. Neonatal systemic candidiasis in a tertiary care centre. Indian Journal of Medical Microbiology 2003;21(1):56-8.

[6] Wadile RG, Bhate VM. Study of clinical spectrum and risk factors of neonatal candidemia. Indian Journal of Pathology and Microbiology 2015;58(4):472-4.

[7] Benjamin DK Jr, Stoll BJ, Fanaroff AA, et al. Neonatal candidiasis among extremely low birth weight infants: risk factors, mortality rates, and neurodevelopmental outcomes at 18 to 22 months. Pediatrics 2006;117(1):84-92.

[8] Jarvis WR. Epidemiology of nosocomial fungal infections, with emphasis on Candida species. Clin Infect Dis 1995;20(6):1526-30.

[9] Shrivastava G, Bajpai T, Bhatambare GS, et al. Neonatal candidemia: clinical importance of species identification. Sifa Medical Journal 2015;2(2):37-40.

[10] Juyal D, Sharma M, Pal S, et al. Emergence of nonalbicans Candida species in neonatal candidemia. North American Journal of Medical Sciences 2013;5(9):541-5.

[11] Tallur SS, Kasturi AV, Nadgir SD, et al. Clinicobacteriological study of neonatal septicaemia in Hubli. The Indian Journal of Pediatrics 2000;67(3):169-74.

[12] Kumar S, Baradkar V, De Mathur AM, et al. A study of neonatal sepsis due to Candida species. Bombay Hospt J 2011;53(1):38-40.

[13] Sardana V, Pandey A, Madan M, et al. Neonatal candidemia: a changing trend. Indian Journal of Pathology and Microbiology 2012;55(1):132-3.
[14] Rao MSS, Surendernath M, Sandeepthi M. Prevalence of neonatal candidemia in a tertiary care institution in Hyderabad, South India. Int J Res Med Sci 2014;2(3):1016-9.

[15] Gupta N, Mittal N, Sood P, et al. Candidemia in neonatal intensive care unit. Ind $\mathrm{J}$ Pathol Microbiol 2001;44(1):45-8.

[16] Juyal D, Kotian S, Sangwan J, et al. Clinicoepidemiological profile, risk factors, and prognosis of neonatal candidemia due to Candida parapsilosis: An emerging threat to neonates. International Journal of Health \& Allied Sciences 2014;3(2):100-4.

[17] Chakrabarti A, Mohan B, Shrivastava SK, et al. Change in distribution \& antifungal susceptibility of Candida species isolated from candidaemia cases in a tertiary care centre during 1996-2000. Indian Journal of Medical Research 2002;116:5-12.

[18] CLSI. Reference method for Broth dilution antifungal susceptibility testing of years, approved standard. CLSI document M27-A3. 3rd edn. Wayne PA: Clinical and Laboratory Standards Institute 2008.

[19] Amboiram P, Balakrishnan U, Ninan B, et al. Incidence of invasive candidal infection in very low birth weight neonates over a period of 5-year: a single institutional study. Surgery 2016;4:15-9.

[20] Yadav S, Dahiya S, Budhani D. Candidemia in neonatal intensive care unit: a cause of concern. Int J Res Med Sci 2017;5(5):2165-7.

[21] Poikonen E, Lyytikäinen O, Anttila VJ, et al. Candidemia in Finland, 1995-1999. Emerging Infectious Diseases 2003;9(8):985-90.

[22] Yadav S, Dahiya S, Budhani D. Candidemia in neonatal intensive care unit: a cause of concern. Int J Res Med Sci 2017;5(5):2165-7.

[23] Banerjee B, Dominic SRM, Baliga S. Clinicomicrobiological study of candidemia in a tertiary care hospital of southern part of India. Iranian Journal of Microbiology 2015;7(1):55-61.

[24] Falagas ME, Roussos N, Vardakas KZ. Relative frequency of albicans and the various non-albicans Candida spp. among candidemia isolates from inpatients in various parts of the world: a systematic review. International Journal of Infectious Diseases 2010;14(11):e954-66.

[25] Sil A, Das A, Konar MC. A study on clinico-mycological profile of systemic fungal infection in neonates: an Indian perspective. IOSR J Dent \& Med Sci 2017;16(5):117-20.

[26] Fu J, Ding Y, Wei B, et al. Epidemiology of Candida albicans and non-C. albicans of neonatal candidemia at a tertiary care hospital in Western China. BMC Infectious Diseases 2017;17(1):329.

[27] Rao S, Ali U. Systemic fungal infections in neonates. J Postgrad Med 2005;51(Suppl 1):S27-9.

[28] Malani A, Hmoud J, Chiu L, et al. Candida glabrata fungemia: experience in a tertiary care center. Clinical Infectious Diseases 2005;41(7):975-81.

[29] Fairchild KD, Tomkoria S, Sharp EC, et al. Neonatal Candida glabrata sepsis: Clinical and laboratory features compared with other Candida species. Pediatr Infect Dis J 2002;21(1):39-43. 
[30] Fidel PL Jr, Vazquez JA, Sobel JD. Candida glabrata: review of epidemiology, pathogenesis and clinical disease with comparison to C. albicans. Clinical Microbiology Reviews 1999;12(1):80-96.

[31] Rani R, Mohapatra NP, Mehta G, et al. Changing trends of Candida species in neonatal septicaemia in a tertiary North Indian hospital. Indian Journal of Medical Microbiology 2002;20(1):42-4.

[32] Panackal AA, Gribskov JL, Staab JF, et al. Clinical significance of azole antifungal drug cross-resistance in
Candida glabrata. Journal of Clinical Microbiology 2006;44(5):1740-3.

[33] Pfaller MA, Diekema DJ. Epidemiology of invasive candidiasis: a persistent public health problem. Clinical Microbiology Reviews 2007;20(1):133-63.

[34] Adhikary R, Joshi S. Species distribution and anti-fungal susceptibility of Candidaemia at a multi super-specialty center in Southern India. Indian Journal of Medical Microbiology
2011;29(3):309-11. 